\title{
Effects of Laser Shock Peening on SCC Behavior of Alloy 600 in Tetrathionate Solution
}

\author{
Abhishek Telang $^{\mathrm{a}^{*}}$, Amrinder S. Gill ${ }^{\mathrm{b}}$, Sebastien Teysseyre ${ }^{\mathrm{c}}$, Seetha R. Mannava ${ }^{\mathrm{a}}$, Dong \\ Qian $^{\mathrm{d}}$ and Vijay K. Vasudevan ${ }^{\mathrm{a}}$ \\ ${ }^{\mathrm{a}}$ Department of Mechanical and Materials Engineering, University of Cincinnati, 2901 \\ Woodside Dr, Cincinnati, OH 45221-0072 \\ ${ }^{\mathrm{b}}$ AK Steel, Research Center, 705 Curtis St, Middletown, OH 45044 \\ ${ }^{\mathrm{c}}$ Idaho National Laboratory, Idaho Falls, ID 83415-2218 \\ ${ }^{\mathrm{d}}$ Department of Mechanical Engineering, University of Texas at Dallas, 800 West Campbell \\ Rd, EC-38, Richardson, TX 75080-3021
}

*Corresponding author information

Abhishek Telang

\section{Mail Address:}

Department of Mechanical and Materials Engineering

University of Cincinnati

415 Rhodes Hall

2901 Woodside Drive, Cincinnati, OH, USA 45221-0012

Email: telangam@mail.uc.edu

Phone: +1 513-556-3119

Fax: +1 513-556-3773

\begin{abstract}
In this study, the effects of laser shock peening (LSP) on stress corrosion cracking (SCC) behavior of nickel based Alloy 600 in tetrathionate solution were investigated. The LSP induced compressive residual stresses and changes in the near surface microstructure, hardness were characterized. The effects of LSP on SCC susceptibility of Alloy 600 in tetrathionate solution were evaluated by slow strain rate tests and constant load tests. The results indicate a significantly longer time to failure and decreased susceptibility to SCC. These improvements were attributed to LSP induced compressive residual stresses, increased yield strength and hardening caused by near-surface microstructural changes.
\end{abstract}


Keywords: A. nickel; B. X-ray diffraction; B. TEM; C. intergranular corrosion; C. stress corrosion

\section{INTRODUCTION}

Alloy 600 has been known to be susceptible to stress corrosion cracking (SCC) at room temperature in polythionic acid environments [1,2] which can be attributed to a combination of susceptible microstructure (sensitization), aggressive species (thiosulfate and tetrathionate) and tensile stresses (applied or residual stresses). Detailed studies on the effect of sensitization treatment, $\mathrm{pH}$, solution concentration, temperature have been used to elucidate the mechanism of intergranular stress corrosion cracking (IGSCC) in polythionic acid environments[3-6]. In general, IGSCC can be mitigated by stabilization heat treatment to modify the microstructure, by changing the environment $(\mathrm{pH}$, reducing the concentration of aggressive species) and by modifying the nature of stresses.

Laser Shock Peening (LSP) is an advanced mechanical surface treatment technique that has been widely used in the aerospace industry to improve component fatigue performance. LSP typically uses a Q-switched Nd:Glass or Nd:YAG $(\lambda=1064 \mathrm{~nm})$ laser of high energy (1-8 J) and short pulse duration (6-20 ns) that passes through a transparent confining medium (water or glass) to ablate a sacrificial thin coating (tape or opaque medium) on the material surface (Figure 1). As the laser beam passes through the water or glass, it is absorbed by the opaque overlay and only a thin layer (few $\mu \mathrm{m}$ ) of this sacrificial layer is ablated creating plasma. Thermal effects on the material surface are suppressed [7]. As the generated plasma continues to absorb the rest of the laser energy, it is readily heated and expands between the material and the confining medium, thus generating a shock wave that propagates through the material. The volume affected by the shock wave is plastically deformed during its propagation to a depth beyond which the peak pressure does not exceed the Hugoniot Elastic limit of the material. The surrounding material in the sub-surface region 
is opposed to the lateral straining resulting in biaxial compressive stresses near and under the surface of the material. Physics of LSP technique have been reported elsewhere $[7,8]$.

Recent interest in life extension of nuclear power plants has contributed to a drive towards mitigation strategies that counter materials degradation challenges in these harsh environments. Fatigue damage from mechanical and/or environmental factors, environmentally assisted cracking (including primary water stress corrosion cracking, intergranular stress corrosion cracking and low temperature crack propagation), and weld residual stresses leading to SCC have been cited as causes of failure in metallic components in service [9]. Surface treatments including laser shock peening, shot peening, water jet peening, low plasticity burnishing have proven to significantly increasing the fatigue resistance of aero alloys, SCC resistance [10] introducing deep compressive residual stresses in the material [11-13]. In theory, the presence of compressive residual stresses should mitigate or perhaps prevent stress corrosion cracking. Shot peening has been generally used to introduce compressive residual stresses to improve fatigue performance and also mitigate stress corrosion cracking [14,15].

In this paper, we present the effects of LSP on the SCC behavior of Alloy 600 in polythionic acids. We first quantified the degree of sensitization using double loop EPR tests and performed detailed characterization of residual stresses, hardness and microstructure after LSP on Alloy 600. Furthermore, slow strain rate tests and constant load tests were used to evaluate the effects of LSP on the IGSCC resistance. The results obtained are described and discussed in detail.

\section{EXPERIMENTAL}

\subsection{Material and Laser Shock Peening}


Alloy 600 plate with 2 mm thickness (Goodfellow Corporation, USA) with chemical composition as listed in Table 1 was used in this study. Heat treatments, LSP parameters and their respective designations are as listed in Table 2.

To characterize the cross section microstructure, mounted samples were ground progressively to 1200 grit, followed by fine polishing with $0.05 \mu \mathrm{m}$ colloidal silica suspension to achieve a mirror finish and etched with $10 \%$ oxalic acid solution (3V, 15s). Microstructure after sensitization and LSP treatments were characterized using a Keyence VH 600 digital optical microscope.

A schematic of the LSP process is shown in Figure 1. LSP was performed using a Qswitched Nd:YAG laser (Continuum Powerlite Plus) with infrared wavelength (1064 nm) and a frequency of $1 \mathrm{~Hz}$. The target (Alloy 600 sample) was moved using a XY table to create a pattern with 50\% overlay. Flowing water was used as confining medium and vinyl tape was used as the sacrificial ablative coating. Based on a series of experiments involving variations in the LSP parameters and counterpart measurements of the surface residual stress, the conditions of pulse energy of 3J, spot size of $2 \mathrm{~mm}$ diameter and pulse width of $20 \mathrm{~ns}$ was used for treatment of all the coupons prior to SCC testing and other characterization. The laser power density for each pulse based on these parameters was $~ 5.6 \mathrm{GW} / \mathrm{cm}^{2}$.

\subsection{Transmission Electron Microscopy}

Characterization of the near-surface regions of the LSP-treated samples is important for understanding the fundamental microstructural changes introduced by these types of surface treatments. Enormous care was given to the preparation of thin foils for Transmission Electron Microscopy (TEM) for observations of the near-surface microstructure of the LSPtreated samples, for which conventional twin-jet electro-polishing is not suitable. Consequently, thin foils from the specimens (sensitized, LSP-treated, etc.) for TEM observations of these microstructural changes were prepared by normal dimpling and ion 
milling procedures. To obtain foils from close to the LSP treated surface, a thin section was sliced off parallel to peened surface, polished to a thickness of $\sim 100 \mu \mathrm{m}$, dimpled using a Fischione Instruments Model 150 dimpler from the side opposite to the peened surface to obtain a one-sided web of thickness of $\sim 20 \mu \mathrm{m}$ near the center, leaving the peened surface untouched and intact. The foil was then ion milled using a Fischione Instruments Model 1010 Ion Milling System only from one side using a shallow incidence angle $\left(12^{\circ}\right)$ and low energy ( $4 \mathrm{keV}, 45^{\circ}$ stage rotation) to ensure that there is no damage induced by ion beam. This enables one to obtain thin areas which are very close to surface. The near surface samples are important as they help in understanding the fundamental microstructural changes introduced by these surface treatments. The same technique was utilized to prepare thin foils of all samples, including those in the non-LSP treated sensitized conditions.

TEM thin foils were observed with a Phillips/FEI CM-20 TEM operated at $200 \mathrm{kV}$ and photographs from relevant regions were recorded under bright field (BF), dark field (DF), weak-beam dark field (WBDF) and selected area diffraction (SAD) modes. Energy dispersive X-ray (EDS) spectroscopy in TEM was conducted at several points at and across grain boundaries to obtain chromium concentration. $\mathrm{X}$ ray intensities were collected at each position for $40 \mathrm{~s}$ by stepping the electron beam (spot size of $15 \mathrm{~nm}$ diameter) in increments of 50-100 nm across grain boundaries in the sensitized (S1 and S2) samples. Standardless analysis was used to quantify the concentration of $\mathrm{Cr}$ and the other elements from the x-ray intensity data.

\subsection{Double Loop Electrochemical Potentiokinetic Reactivation (DLEPR) tests}

Electrochemical potentiokinetic reactivation (EPR) tests have been used previously to quantify degree of susceptibility to IGA/IGSCC in Ni-based Alloy $600[16,17]$. The double loop electrochemical potentiokinetic (DLEPR) test is a fast, reproducible and quantitative method to determine degree of sensitization (DOS). The DOS after heat treatments was 
quantified as the ratio of maximum current in the reactivation loop to maximum current in the activation loop. DLEPR tests were performed in accordance with ASTM G108-94 in a solution composed of $0.01 \mathrm{M} \mathrm{H}_{2} \mathrm{SO}_{4}+20$ ppm KSCN using a Gamry Potentiostat (Reference 600) [16]. The scan rate was set at $0.5 \mathrm{mV} / \mathrm{s}$ for activation and reactivation loop and the sample size was $1 \mathrm{~cm}^{2}$. Each heat treated coupon was mechanically ground to 1200 grit, wet polished with $1 \mu \mathrm{m}$ diamond suspension and finished with $0.05 \mu \mathrm{m}$ colloidal silica suspension. Freshly prepared solution was de-aerated with high purity Ar gas before and during each test. All tests were performed at room temperature. Samples were kept immersed in the test solution for 1 hour at open circuit potential before the start of each test. The extent of intergranular attack after DLEPR tests was observed using a FEI XL-30 Scanning Electron Microscope (SEM).

\subsection{Residual Stress and Hardness Characterization}

In the $\sin ^{2} \psi$ method, a number of XRD measurements are made at different psi ( $\left.\psi\right)$ tilts where $\psi$ is the angle between the normal of the sample and the normal of the diffracting plane. Presence of strain causes peak shift (2-theta peak position) and inter-planar spacing (d) can be measured at each $\psi$ tilt. The stress was then calculated by fitting the data in the d versus $\sin ^{2} \psi$ plot and knowledge of the plane-specific x-ray diffraction elastic constants (S1 and S2) of the material[18].

Residual stresses induced in the material after LSP treatment were measured using the $\sin ^{2} \psi$ technique with a Proto LXRD instrument (single axis goniometer using $\Omega$ geometry) with parameters as listed in Table 3. Calibration of the system was checked by collecting a diffraction pattern from a strain free standard polycrystalline SS316 powder prior to conducting the experiment in accordance with ASTM E915-10 ("Verifying the Alignment of X-ray Diffraction Instrumentation for Residual Stress Measurement”) [19]. 
To measure residual stress through depth, samples were electro-polished to remove 10 - 50 $\mu$ m layers using 87.5:12.5 vol.\% $\mathrm{CH}_{3} \mathrm{OH}: \mathrm{H}_{2} \mathrm{SO}_{4}$ solution. Thickness of layer removed was calculated by measuring the thickness of the sample with a precise micrometer before and after electro-polishing. Strain and layer removal corrections were applied to the residual stress values using established procedures included in the software.

The relaxation of LSP induced residual stresses due to applied stress was observed by measuring residual stresses on the gage section of the same sample before and after constant load SCC tests. Before testing, residual stresses were measured at least 2 times along and perpendicular to the loading direction on each sample. After constant load tests, residual stresses were measured twice on the gage section of samples that did not fail in 500 hours. The average values of surface residual stresses on the gage section along the loading direction have been reported in this study.

Hardness measurements were performed using a CSM Instruments Nano-Micro Indentation system with a Berkovich indenter, a load of $100 \mathrm{mN}$ with loading/unloading rate of $200 \mathrm{mN} / \mathrm{min}$ and a pause of 5 seconds at the peak load. At least 3 hardness values were recorded at each depth on polished cross-sectioned LSP treated samples.

\subsection{SCC tests}

\section{a. Slow Strain Rate Test (SSRT)}

Flat samples with the dimensions as shown in Figure 2 were machined using wire EDM from sensitized sheets of Alloy 600, polished to 1200 grit, degreased with acetone, dried and immersed in the test solution for 1 hour prior to straining. Samples were strained at rates of $2 \times 10^{-6} / \mathrm{s}$ and $10^{-6} / \mathrm{s}$ for S1 and S2 sensitization conditions respectively. LSP was performed using vinyl tape as the ablative layer and water as the confining medium with the parameters described earlier. Samples were LSP treated on 2 opposite sides of the gage section as shown in Figure 2. Slow strain rate tests (SSRT) were performed at room 
temperature with a mechanical testing system (Cortest Inc. Willoughby, Ohio) driven by a servo motor and fitted with a custom built environmental chamber. Load and displacement values were recorded periodically using calibrated load cells and 2 LVDT's respectively and samples were tested to failure. Strain was calculated from the average displacement measured by the 2 LVDT's. Solutions with concentration $0.001 \mathrm{M}$ or $0.005 \mathrm{M}$ were prepared using reagent grade $\mathrm{Na}_{2} \mathrm{~S}_{4} \mathrm{O}_{6}$ sodium tetrathionate (Sigma-Aldrich) and distilled water. For certain SSRT tests, the solution $\mathrm{pH}$ was adjusted to 3 with the appropriate amount of dilute $\mathrm{H}_{2} \mathrm{SO}_{4}$.

\section{b. Constant Load Tests}

Constant load tests were performed by loading the samples (dimensions as shown in Figure 2) using calibrated Proof rings (Cortest Inc. Willoughby, Ohio) to the predetermined loads/stresses and the time to failure was recorded using a timer switch. The test solutions were prepared according to the same procedure described earlier. Fracture surfaces of failed samples from SSRT and constant load SCC tests were observed in an FEI XL-30 SEM to ascertain the mode of failure.

\section{RESULTS}

In this section, we first present the effects of LSP on the microstructure, residual stresses and hardness. Next, SCC test results including SSRT and constant load tests in tetrathionate solution are described.

\subsection{Microstructure}

The cross-section optical micrographs recorded in Figure 3(a,b) from the untreated (S1) and LSP-treated (LSP1) samples respectively show no changes in the grain size following the LSP treatment. Figure 3(c) shows the microstructure of the sensitized sample in which a small area was LSP treated. No significant difference in grain size can be observed between the untreated and LSP-treated regions in the micrograph. Other studies on effects of 
LSP on microstructure have reported no significant changes in grain size due to the relatively lower plastic strain induced during the treatment [20].

Heat treatment conditions were chosen such that Alloy 600 was sensitized to induce IGSCC susceptibility in tetrathionate solutions at room temperature. TEM micrographs from the sensitized samples (S1 and S2) showing in carbide precipitation along grain boundaries can be observed in Figure 4(a, b) respectively. Carbides $\left(\mathrm{M}_{7} \mathrm{C}_{3}, \mathrm{M}_{23} \mathrm{C}_{6}\right)$ along the grain boundaries usually results along the grain boundaries usually results in depletion of Cr from the surrounding regions, the extent of which depends on the sensitization treatment. Chromium concentration profiles across grain boundaries in sensitized samples (S1 and S2) are shown in Figure 4(c). Cr concentration was as low as 9.4 wt.\% and $13.4 \mathrm{wt} . \%$ at the grain boundary in S1 and S2 respectively. This suggests that precipitation of carbides during both sensitization treatments led to Cr depletion.

LSP being a mechanical surface treatment technique influences the microstructure and TEM micrographs from the near surface region after LSP treatment have been presented in Figure 5(a-f). The pressure pulse/shock wave generated during the LSP treatment causes plastic deformation, which results in the formation of a high dislocation density and misoriented sub-grains/crystallites that have sizes in the range of $30-200 \mathrm{~nm}$ in the nearsurface regions, which can be clearly seen in the BF micrographs in Figure 5(a-c). In addition, narrow twins were observed occasionally as seen in the BF micrograph in Figure 5(d) and confirmed by the extra twin reflections at the expected positions for FCC crystals in the [011] SAD pattern in Figure 5(e) from the encircled area surrounding the twins and revealed more clearly in the DF micrograph in Figure 5(f) recorded from a twin reflection. This is in accordance with other studies that have reported a high dislocation density and twinning after shot peening and LSP treatments [7,11, 21].

\subsection{Residual stresses, FWHM and hardness}


Residual stresses versus distance from the peened surface, measured using the $\sin ^{2} \psi$ method in two orthogonal directions ( $\mathrm{X}$ and $\mathrm{Y}$ ), are shown in Figure 6. The residual stresses were of the order of about $-500 \mathrm{MPa}$ at the surface and the depth of the compressive residual stress layer was about $0.4 \mathrm{~mm}$ (in a $2 \mathrm{~mm}$ thick sample). The surface residual stresses have been significantly modified from tensile (100 MPa) before LSP to compressive (-500 MPa) after LSP.

X-ray diffraction peak broadening characterized by full widths at half maximum (FWHM) values, which are a qualitative indication of the level of plastic strain introduced in a material were also recorded during residual stress measurements and have been plotted in Figure 7. In LSP treated Alloy 600, the level of plastic strain is higher at the surface (FWHM of $~ 3.5$ ) but drops rapidly in the first $100 \mu \mathrm{m}$ and decreases gradually through depth to a more or less constant value after $\sim 400 \mu \mathrm{m}$.

Indentation hardness at the surface after LSP was about 4.99 GPa and drops gradually to $3.75 \mathrm{GPa}$ at $600 \mu \mathrm{m}$ below the surface. The indentation hardness before LSP was about 3.28 GPa and the increase in hardness can be attributed to the repeated impacts during the LSP process. Pressure pulse generated during LSP process have short pulse duration and attenuates as it travels through the material. This explains the gradient nature of residual stresses, plastic deformation and hardness.

\subsection{Double Loop Electrochemical Potentiokinetic Reactivation Tests}

The DOS values obtained from DLEPR for solution annealed and sensitized samples are shown in Table 4 . The sensitization treatment at $650^{\circ} \mathrm{C}$ resulted in increased DOS from 0.56 for AR to 0.78 and 2.04 for S2 and S1 conditions respectively.

A higher degree of sensitization indicates a deeper Cr depletion zone and greater the susceptibility to intergranular stress corrosion cracking. SEM micrographs of the surface after DLEPR tests for AR, S1 and S2 samples are shown in Figure 8(a-c) respectively. The 
increased attack on the grain boundaries can be clearly seen in microstructure of the sensitized samples and is indicative of the amount of $\mathrm{Cr}$ depletion due to the sensitization treatment, which is also consistent with the Cr concentration profiles in Figure 4 determined using EDS.

\subsection{Slow Strain Rate Tests (SSRT)}

SSRTs were used to quantify the susceptibility of sensitized Alloy 600 to IGSCC in tetrathionate solution at room temperature and particularly to understand the effects of LSP on the SCC susceptibility. Figure 9 shows the effect of sensitization treatments (different DOS), solution concentration and LSP on the SCC susceptibility in tetrathionate solution. Strain to failure, maximum stress and mode of failure from SSR tests conducted in this study are tabulated in Table 5. For comparison, sets of untreated (S1) and LSP treated (LSP1) Alloy 600 (same sensitization condition) were strained at the same rate in the same environment. Both S1 and S2 sensitization conditions were observed to be susceptible to IGSCC in the $0.001 \mathrm{M}$ tetrathionate solution. Maximum elongations were 31\% and $23 \%$ for S1 and S2 respectively in the tetrathionate solution $(0.001 \mathrm{M})$ as compared to $\sim 60 \%$ in air (neutral environment). DLEPR test results (Table 4) and Cr depletion profiles (Figure 4) indicated that S2 was less sensitized as compared to S1 and hence a lower strain rate was used for SSR tests. This ensured that the mode of failure was intergranular SCC. Once the baseline conditions (DOS, strain rate and environment) were established, LSP treated samples were tested under the same conditions. Since the LSP treatment was done after sensitization treatment, any changes can be attributed to the effects of LSP. The stress-strain curves shown in Figure 9 indicate higher yield stress (YS) and ultimate tensile strength (UTS) for LSP treated Alloy 600 compared with the untreated condition. This effect was seen in all LSP treated samples irrespective of the sensitization condition or environment. For example, YS 
was $\sim 40 \%$ higher after LSP for the same sensitization condition. The strain to failure was also higher for LSP treated samples compared with untreated samples.

While SSRTs can be used to provide information about the susceptibility of an alloy in the given environment, constant/cyclic load tests are more representative of the stress state. Also, the residual stresses induced by LSP are likely to relax on straining beyond the yield stress of the material. The increase in yield stress and ultimate tensile strength after LSP would possibly increase the threshold applied stress for IGSCC in a given environment. A single crack that propagated through the cross-section of the sample was observed in all samples that were tested in $0.001 \mathrm{M} \mathrm{Na}_{2} \mathrm{~S}_{4} \mathrm{O}_{6}$ solution. This may be attributed to a combination of less sensitization, less aggressive environment and strain rate. Secondary cracks were observed in samples that were tested in the acidified (pH 3) $0.001 \mathrm{M} \mathrm{Na}_{2} \mathrm{~S}_{4} \mathrm{O}_{6}$ solution thereby indicating that the acidified solution was more aggressive towards sensitized Alloy 600. Low magnification SEM micrographs of fracture surfaces of failed samples after SSRTs have been shown in Figure 10 (a, c, e and g) whereas the higher magnification images in Figures 10 (b, d, $\mathrm{f}$ and h) show inter granular cracks in more detail. The mode of failure was (partial) IGSCC for sensitized and LSP treated samples.

\subsection{Constant Load Tests}

Constant load tests were used to evaluate the effects of LSP on Alloy 600 under static loading conditions in different solution concentrations. Figure 11 summarizes the effects of LSP on SCC resistance in terms of applied stress and time to failure. For the untreated samples, the time to failure decreased with increase in applied stress in $0.001 \mathrm{M}$ tetrathionate solution. The applied loads were chosen such that they were between $80-140 \%$ of the YS load for sensitized Alloy 600. In general, LSP treated samples tested at same loads had much longer time to failure or did not fail in 500 hours after which the tests were terminated. Sensitized samples failed in shorter time compared with the LSP treated samples for all loads and solution concentrations/pH tested. 
The increase in time to failure after LSP can be attributed to the beneficial compressive residual stresses (Figure 6) induced by LSP which were as high as -550 MPa at the surface at the start of test, as well as near-surface hardening (Figure 7) caused by the increased dislocation density and formation of misoriented, 30-100 nm-size subgrains/crystallites, whose boundaries act as obstacles to dislocation glide (Figure 5). Cracks were not observed in sensitized Alloy 600 (S1) after 500 hours in $0.001 \mathrm{M}$ tetrathionate solution at a load of $300 \mathrm{MPa}$ which suggests a stress threshold for IGSCC. SEM micrographs of the fracture surfaces from constant load tests for sensitized (S1) and LSP treated (LSP1) are shown in Figure 12(a, b) respectively, and intergranular cracking was observed in both cases.

The effects of LSP on resistance to IGSCC in acidified tetrathionate solution (0.001 $\mathrm{M} \mathrm{Na}_{2} \mathrm{~S}_{4} \mathrm{O}_{6} \mathrm{pH}$ 3) were also investigated. The time to failures dramatically decreased for sensitized samples (S1) from 176 hours in $0.001 \mathrm{M}$ tetrathionate to 30 hours when the $\mathrm{pH}$ was reduced to 3. In this test, the LSP treated sample showed multiple cracks in the gage section after 423 hours (applied stress $=350 \mathrm{MPa})$. At a lower applied stress $(300 \mathrm{MPa})$, the LSP treated sample did not show cracking even after 500 hours, whereas the untreated sample failed in about 45 hours in the acidified tetrathionate solution.

\section{DISCUSSION}

The SSRT and constant load test results suggest that LSP has a significant effect on improving the IGSCC resistance of Alloy 600. In this section, SSRT data were used to calculate the SCC susceptibility index and quantify the effect of LSP induced residual stresses. In addition, we discuss the effects of LSP under different applied load, solution concentration and DOS conditions, with attention to mechanistic aspects.

\subsection{SCC Susceptibility Index}


To quantify the effect of LSP on the susceptibility to IGSCC in tetrathionate solutions, the following equation (Equation 1) proposed by Abe et al. [21] was used to calculate the SCC susceptibility index ( $\left.\mathrm{I}_{\mathrm{SCC}}\right)$,

$$
I_{S C C}=\left[\frac{1+e_{n}}{1+e_{n}^{S C C}}\right] \times\left[\frac{P_{n}}{P_{n}^{S C C}}-1\right]
$$

where $e_{n}$ and $e_{n} S C C$ are strains at the maximum load for load-elongation plot in air and environment respectively. $P_{n}$ and $P_{n} S C C$ are the maximum loads for load-elongation plot in air

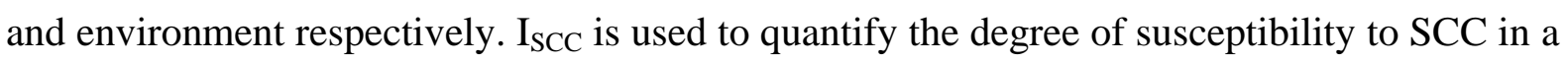
particular solution with respect to a neutral environment at the same strain rate. Table 6 summarizes the $\mathrm{I}_{\mathrm{SCC}}$ for the tests as per SCC susceptibility proposed by Abe et al.[22].

SCC susceptibility of LSP treated sensitized Alloy 600 was lower than that of counterpart untreated sensitized Alloy 600. Increasing the solution concentration from $0.001 \mathrm{M}$ to $0.005 \mathrm{M} \mathrm{Na}_{2} \mathrm{~S}_{4} \mathrm{O}_{6}$ did not increase the $\mathrm{I}_{\mathrm{SCC}}$ appreciably but decreasing the $\mathrm{pH}$ to 3 had a more significant effect. S2 was strained at a slower rate than S1 which explains the relatively higher susceptibility to SCC in the same solution despite having lower DOS. The higher yield stress and UTS contributed to the reduced SCC susceptibility for LSP samples. Iscc was lower even in more aggressive environment where the $\mathrm{pH}$ was 3. SSRT tests involve straining the samples continuously and residual stresses relax as the applied stress exceeds the yield stress [18]. Hence, residual stresses are likely to be ineffective in mitigating SCC if the samples are plastically deformed to large strains.

As noted earlier, the mode of failure was IGSCC even for the LSP treated samples which have lower $\mathrm{I}_{\mathrm{SCC}}$. It is important to note that the LSP treated samples show a much higher yield stress as compared with the untreated samples. This increase in yield stress is a result of LSP induced deep compressive residual stresses and near surface hardening from the increased dislocation density and refined sub-grains/crystallites, whose boundaries act as obstacles to dislocation glide. $\mathrm{I}_{\mathrm{scc}}$ proposed by Abe at al. takes into account the strain, load at 
the maximum load and is better suited to quantify the effects of residual stresses on SCC susceptibility. The higher yield stress for LSP1 vs S1 and LSP2 vs S2 contribute to reduced susceptibility.

\subsection{Effect of Applied Load and Solution Concentration}

Constant load test results presented in Figure 11 suggest that lower degree of sensitization increased the time to failure in the same solution under similar applied loads. Residual stresses of $\sim-150 \mathrm{MPa}$ were measured after the constant load test (applied stress $=$ 400MPa) in cases where samples did not fail in 500 hours. This is lower than the initial surface residual stresses of $\sim-550 \mathrm{MPa}$ before testing. This indicates that a part of the residual stresses relaxed during loading. It is known that residual stresses will redistribute and relax as cyclic/static load is applied especially if it exceeds the yield stress of the material [23]. This was confirmed by measuring residual stresses on the gage section constant load SCC tests and the results have been summarized in Table 7. Also, residual stresses were close $\sim 10 \mathrm{MPa}$ on LSP treated samples that had failed before 500 hours. This indicates that compressive residual stresses induced by LSP had a significant effect in the mitigating SCC. Moreover, this effect was seen in cases where the solution is more acidic ( $\mathrm{pH} 3)$. The presence of compressive residual stresses (albeit lower than those in the as LSP treated condition) after constant load tests (500 hours) suggests that threshold stress was higher.

Constant stress tests on the sensitized, but non-LSP treated samples were conducted at stress levels both below and above the yield stress. At applied stresses below the yield stress, for example $300 \mathrm{MPa}$, failure due to SCC was not observed in the normal $\mathrm{pH}$ solution but occurred at short times in the more aggressive environment ( $\mathrm{pH} 3$ ). The applied stress was close to or above the yield stress of the sensitized samples but below the yield stress of LSP treated samples. It must be noted that this increase in threshold stress may be lower or higher in more or less aggressive solution/sensitization conditions respectively. 
Tests at stresses above the yield stress of the LSP-treated samples were not conducted because plastic yielding would cause relaxation of the compressive residual stresses and little or no improvement in SCC life would be expected. Nevertheless, the important conclusion to be drawn is that LSP considerably increases the threshold stress and life for failure from SCC. The better resistance of LSP treated samples to SCC under constant load in tetrathionate solutions may be attributed to the higher yield stress (from the hardening associated with the increased dislocation density and refined sub-grains/crystallites whose boundaries serve as obstacles to slip).

Tsai et al. [14] had observed increased resistance of shot peened sensitized Alloy 600 to SCC in thiosulfate solutions and the beneficial effect was attributed to the presence of compressive residual stresses though the residual stresses were not quantified. Sano et al. [8] also observed increased resistance to crack initiation after LSP treatment on SS304 in high temperature pure water. They observed that LSP (without coating) prevented SCC even if the residual stresses introduced after LSP were as low as -100 MPa. In addition, LSP had prevented crack initiation and mitigated crack propagation. Peyre et al. [24] reported that LSP acted like a tensile stress inhibitor which contributed to the improved SCC resistance in boiling $\mathrm{MgCl}_{2}$ solution in SS316L. Recently, Lu et al. [25] attributed the increase in SCC resistance of ANSI 304 austenitic stainless steel in boiling $\mathrm{MgCl}_{2}$ solution to modified grain refinement and residual stresses due to LSP treatment. Lu and colleagues also observed relaxation of residual stresses after LSP treated AISI 304 SS samples were bent to form Ubend specimen [26]. Additionally, these samples failed by SCC in boiling $\mathrm{MgCl}_{2}$ solution as compared with samples that were LSP treated after being bent into U- bend specimens that did not fail. This indicates that LSP induced compressive residual stresses significantly reduce the susceptibility of austenitic stainless steels to SCC. Results from this study suggest 
that compressive residual stresses and near surface hardening induced by LSP treatment substantially increased the resistance of alloy 600 to SCC in tetrathionate solution.

\section{CONCLUSIONS}

The findings of this study have led to the following conclusions.

1. DLEPR tests were used to quantify the DOS for sensitized Alloy 600. Sensitized Alloy 600 was susceptible to IGSCC in sodium tetrathionate solution at room temperature. A higher DOS corresponded to greater susceptibility of sensitized Alloy 600 to SCC in tetrathionate solution.

2. Deep compressive residual stresses were introduced and a substantial increase in hardness and strength was observed after LSP in Alloy 600. Near-surface microstructure of LSP treated Alloy 600 shows deformation bands, high dislocation densities, misoriented subgrains of 30-200 nm size and occasionally narrows twins.

3. Slow strain rate tests used to evaluate IGSCC susceptibility of sensitized Alloy 600 in tetrathionate solution revealed that LSP substantially reduced the susceptibility of sensitized Alloy 600 to IGSCC for conditions with different DOS, solution concentration and $\mathrm{pH}$.

4. LSP treatment led to a significant improvement in resistance to SCC indicated by longer time to failure or no failure at same applied stress in sensitized alloy 600 as shown by constant load tests for different DOS and pH in tetrathionate solutions.

5. The improvements in SCC resistance by LSP treatment are attributed to a combination of deep compressive residual stresses, near-surface microstructural changes and the associated hardening and increased yield strength of the alloy.

\section{ACKNOWLEDGEMENTS}


The authors are grateful for financial support of this research by the Nuclear Energy University Program (NEUP) of the US Department of Energy contract \#102835 issued under prime contract DE-AC07-05ID14517 to Battelle Energy Alliance, LLC. We also gratefully acknowledge the contribution of the State of Ohio, Department of Development and Third Frontier Commission, which provided funding in support of "Ohio Center for Laser Shock Processing for Advanced Materials and Devices” equipment in the Center that was used in this work. The authors are also grateful for the support of David Simmermon (Cincinnati State Technical and Community College) for LSP setup. Any opinions, findings, conclusions, or recommendations expressed in these documents are those of the author(s) and do not necessarily reflect the views of the DOE, State of Ohio, Department of Development.

\section{REFERENCES}

[1] D. Van Rooyen, Review of the stress corrosion cracking of Inconel 600, Corrosion. 31 (1975) 327-337.

[2] R. Bandy, D. Van Rooyen, Effect of Thermal Stabilization on the Low Temperature Stress Corrosion Cracking of Inconel 600, Corrosion. 40 (1984) 281-289.

[3] J. Kai, C. Tsai, T. Huang, M. Liu, The effects of heat treatment on the sensitization and SCC behavior of INCONEL 600 alloy, Metall. Trans. A. 20 (1989) 1077-1088.

[4] S. Hsu, S. Tsai, J. Kai, C. Tsai, SCC behavior and anodic dissolution of Inconel 600 in low concentration thiosulfate, J. Nucl. Mater. 184 (1991) 97-106.

[5] R. Bandy, R. Roberge, R. Newman, Low temperature stress corrosion cracking of Inconel 600 under two different conditions of sensitization, Corros. Sci. 23 (1983) 995-1006.

[6] R.C. Newman, R. Roberge, R. Bandy, Environmental Variables in the Low Temperature Stress Corrosion Cracking of Inconel 600, Corrosion. 39 (1983) 386-390.

[7] P. Peyre, R. Fabbro, Laser shock processing a review of the physics and applications, Opt. Quantum Electron. 27 (1995) 1213-1229.

[8] Y. Sano, Y. Sakino, N. Mukai, M. Obata, I. Chida, T. Uehara, M. Yoda, Y-C Kim , Laser Peening without Coating to Mitigate Stress Corrosion Cracking and Fatigue Failure of Welded Components, Mater. Sci. Forum. 580-582 (2008) 519-522.

[9] S.J. Green, Methods for preventing steam generator failure or degradation, Int. J. Press. Vessel. Pip. 25 (1986) 359-391. 
[10] P. Peyre, X. Scherpereel, L. Berthe, C. Carboni, R. Fabbro, G. Be, Surface modifications induced in 316L steel by laser peening and shot-peening . Influence on pitting corrosion resistance, Mater. Sci. Eng. A. 280 (2000) 294-302.

[11] A. Gill, A. Telang, S.R. Mannava, D. Qian, Y.-S. Pyoun, H. Soyama, Vijay K. Vasuedevan, Comparison of Mechanisms of Advanced Mechanical Surface Treatments in Nickel-Based Superalloy, Mater. Sci. Eng. A. (2013).

[12] C.S. Montross, T. Wei, L. Ye, G. Clark, Y. Mai, Laser shock processing and its effects on microstructure and properties of metal alloys : a review, Int. J. Fatigue. 24 (2006) 1021-1036.

[13] C. Ye, A. Telang, A.S. Gill, S. Suslov, Y. Idell, K. Zweiacker, Zhong Zhou, Dong Qian, S.R.Mannava, Vijay K. Vasuedevan, Gradient nanostructure and residual stresses induced by ultrasonic nano-crystal surface modification in 304 austenitic stainless steel for High strength and High ductility, Mater. Sci. Eng. A. 613 (2014) 274-288.

[14] W. Tsai, C. Chang, J. Lee, Effects of shot peening on corrosion and stress corrosion cracking behaviors of sensitized alloy 600 in thiosulfate solution, Corrosion. 50 (1994) 98-105.

[15] P. Hurrell, D. Everett, A. Gregg, S. Bate, Review of residual stress mitigation methods for application in nuclear power plant, Proc. ASME Press. Vessel. Pip. Conf. 6 (2006) 801-812.

[16] Y. Lim, H. Kim, J. Han, J. Kim, H. Kwon, Influence of laser surface melting on the susceptibility to intergranular corrosion of sensitized Alloy 600, Corros. Sci. 43 (2001) 13211335.

[17] M. Ahn, H. Kwon, J. Lee, Predicting Susceptibility of Alloy 600 to Intergranular Stress Corrosion Cracking Using a Modified Electrochemical Potentiokinetic Reactivation Test, Corros. Sci. 51 (1995) 441-449.

[18] P.J. Withers, H.K.D.H. Bhadeshia, Residual stress Part 1 - Measurement techniques, Mater. Sci. Technol. 17 (2001) 355-365.

[19] ASTM E915-10, Standard Test Method for Verifying the Alignment of X-Ray Diffraction Instrumentation for Residual Stress, ASTM International, West Conshohocken, 2010.

[20] Y. Sano, M. Obata, T. Kubo, N. Mukai, M. Yoda, K. Masaki, Y. Ochi, Retardation of crack initiation and growth in austenitic stainless steels by laser peening without protective coating, Mater. Sci. Eng. A. 417 (2006) 334-340.

[21] I. Nikitin, I. Altenberger, Comparison of the fatigue behavior and residual stress stability of laser-shock peened and deep rolled austenitic stainless steel AISI 304 in the temperature range 25-600² Mater. Sci. Eng. A. 465 (2007) 176-182.

[22] S. Abe, M. Kojima, Y. Hosoi, Stress Corrosion Cracking Susceptibility Index, ISCC, of Austenitic Stainless Steels in Constant Strain-Rate Test (STP 665), STP 665 Stress Corrosion Cracking-The Slow Strain-Rate Technique (ASTM International), in: 1979: pp. 294-304.

[23] B.L. Boyce, X. Chen, J.O. Peters, J.W. Hutchinson, R.O. Ritchie, Mechanical relaxation of localized residual stresses associated with foreign object damage, Mater. Sci. Eng. A. 349 (2003) 48-58. 
[24] P. Peyre, L. Berthe, R. Fabbro, Corrosion reactivity of laser-peened steel surfaces, J. Mater. Eng. Perform. 9 (2000) 656-662.

[25] J.Z. Lu, H. Qi, K.Y. Luo, M. Luo, X.N. Cheng, Corrosion behaviour of AISI 304 stainless steel subjected to massive laser shock peening impacts with different pulse energies, Corros. Sci. 80 (2014) 53-59.

[26] J.Z. Lu, K.Y. Luo, D.K. Yang, X.N. Cheng, J.L. Hu, F.Z. Dai, H. Qi, L. Zhang,J. S. Zhong,Q.W.Wang, Y.K. Zhang, Effects of laser peening on stress corrosion cracking (SCC) of ANSI 304 austenitic stainless steel, Corros. Sci. 60 (2012) 145-152. 


\begin{tabular}{ccccccccccccc}
\hline $\mathbf{C}$ & $\mathbf{M n}$ & $\mathbf{S i}$ & $\mathbf{S}$ & $\mathbf{C r}$ & $\mathbf{F e}$ & $\mathbf{C o}$ & $\mathbf{C d}$ & $\mathbf{T i}$ & $\mathbf{C u}$ & $\mathbf{P}$ & Al & $\mathbf{N i}$ \\
\hline 0.08 & 0.16 & 0.18 & $\begin{array}{c}0.001 \\
\text { max. }\end{array}$ & 14.99 & 8.05 & 0.18 & 0.01 & 0.18 & 0.1 & $\begin{array}{c}0.001 \\
\text { max. }\end{array}$ & 0.08 & Bal. \\
\hline
\end{tabular}

Table 1. Chemical composition of the Inconel Alloy 600 used in this study.

\begin{tabular}{cc}
\hline Designation & Sensitization and LSP treatments \\
\hline AR & As Received (annealed) \\
S1 & $\mathrm{AR}+650^{\circ} \mathrm{C}, 5 \mathrm{~h}$, air cooled \\
S2 & $\mathrm{AR}+650^{\circ} \mathrm{C}, 2 \mathrm{~h}$, air cooled \\
LSP1 & $\mathrm{S} 1+\mathrm{LSP} 5.6 \mathrm{GW} / \mathrm{cm}^{2}$ \\
LSP2 & $\mathrm{S} 2+\mathrm{LSP} 5.6 \mathrm{GW} / \mathrm{cm}^{2}$ \\
\hline
\end{tabular}

Table 2. Designation for sensitization and LSP treatments considered in this study.

\begin{tabular}{ll}
\hline Item & Description \\
\hline Detector & Position sensitive scintillation detector(PSSD) $20^{\circ} 2 \theta$ \\
Radiation & $\mathrm{MnK \alpha}_{1}\left(\lambda=2.10314 \mathrm{~A}^{\circ}\right)$ \\
Tilt angles & $0, \pm 2.58, \pm 9.07, \pm 12.45, \pm 18.8, \pm 23.0$ \\
Aperture size & $1 \mathrm{~mm}$ diameter \\
Plane(Bragg's angle) & $\{311\}, 156^{\circ}$ \\
X-ray elastic constant & $\mathrm{S} 2 / 2: 5.66 \times 10^{-6} \mathrm{MPa}^{-1}$ \\
\hline
\end{tabular}

Table 3. XRD parameters used for residual stress measurement

\begin{tabular}{ccc}
\hline Designation & DOS, \% & $\begin{array}{c}\text { Sensitization time at } \\
\mathbf{6 5 0} \mathbf{C}, \mathbf{h}\end{array}$ \\
\hline AR & $0.56 \pm 0.06$ & 0 \\
S1 & $2.04 \pm 0.09$ & 5 \\
S2 & $0.78 \pm 0.08$ & 2 \\
\hline
\end{tabular}

Table 4. Degree of sensitization for the test conditions considered in this study.

\begin{tabular}{|c|c|c|c|c|c|}
\hline Specimen/Environment & YS, MPa & UTS, MPa & Elongation, \% & Test variable & Mode of Failure \\
\hline S1_0.001M & 280 & 514 & 31.27 & Baseline & IGSCC \\
\hline LSP1_0.001M & 528 & 731 & 31.14 & LSP & IGSCC \\
\hline S1_0.005M & 310 & 514 & 33.82 & Solution concentration & IGSCC \\
\hline LSP1_0.005M & 554 & 725 & 41.83 & $\begin{array}{c}\text { LSP,Solution } \\
\text { concentration }\end{array}$ & IGSCC \\
\hline
\end{tabular}




\begin{tabular}{|c|c|c|c|c|c|}
\hline S2_0.001M & 296 & 441 & 23.10 & \%DOS, strain rate & IGSCC \\
\hline LSP2_0.001M & 520 & 787 & 42.50 & $\begin{array}{c}\text { \%DOS, strain rate, } \\
\text { LSP }\end{array}$ & IGSCC \\
\hline S1_0.001M_pH3 & 312 & 496 & 18.89 & Solution pH & IGSCC \\
\hline LSP1_0.001M_pH3 & 563 & 808 & 29.08 & Solution pH, LSP & IGSCC \\
\hline S1_Air & 330 & 864 & 66.78 & Neutral environment & Ductile \\
\hline S2_Air & 326 & 753 & 61.47 & Neutral environment & Ductile \\
\hline
\end{tabular}

Table 5. Summary of all results from SSR tests on sensitized Alloy 600

\begin{tabular}{cccc}
\hline Specimen/Environment & Maximum load, N & $\begin{array}{c}\text { Strain at maximum } \\
\text { load, \% }\end{array}$ & I SCC \\
\hline S1_0.001M & 359 & 16.58 & 0.88 \\
LSP1_0.001M & 510 & 15.02 & 0.24 \\
S1_0.005M & 359 & 17.82 & 0.87 \\
LSP1_0.005M & 506 & 12.82 & 0.26 \\
S2_0.001M & 308 & 10.58 & 1.31 \\
LSP2_0.001M & 549 & 29.93 & 0.11 \\
S1_0.001M_pH3 & 346 & 12.33 & 1.00 \\
LSP1_0.001M_pH3 & 564 & 22.61 & 0.09 \\
S1-Air & 603 & 50.90 & - \\
\hline
\end{tabular}

Table 6. Comparison of SCC susceptibility index using strain and maximum load relationship for sensitized and LSP treated Alloy 600.

\begin{tabular}{cccc}
\hline $\begin{array}{c}\text { Sample Designation, } \\
\text { Environment }\end{array}$ & $\begin{array}{c}\text { Applied stress for } \\
\text { 500 hours, MPa }\end{array}$ & $\begin{array}{c}\text { Residual stress } \\
\text { before test, MPa }\end{array}$ & $\begin{array}{c}\text { Residual stress } \\
\text { after test, MPa }\end{array}$ \\
\hline LSP1, 0.001 M & 350 & $-550 \pm 15$ & $-156 \pm 14$ \\
LSP2, 0.001 M & 400 & $-550 \pm 15$ & $-172 \pm 18$ \\
LSP1, 0.001 M & 500 & $-550 \pm 15$ & $-14 \pm 10$ \\
LSP1, 0.001M pH 3 & 300 & $-550 \pm 15$ & $-165 \pm 17$ \\
\hline
\end{tabular}

Table 7. Average value of surface residual stresses on the gage section along the loading direction after constant load tests in tetrathionate solution. 


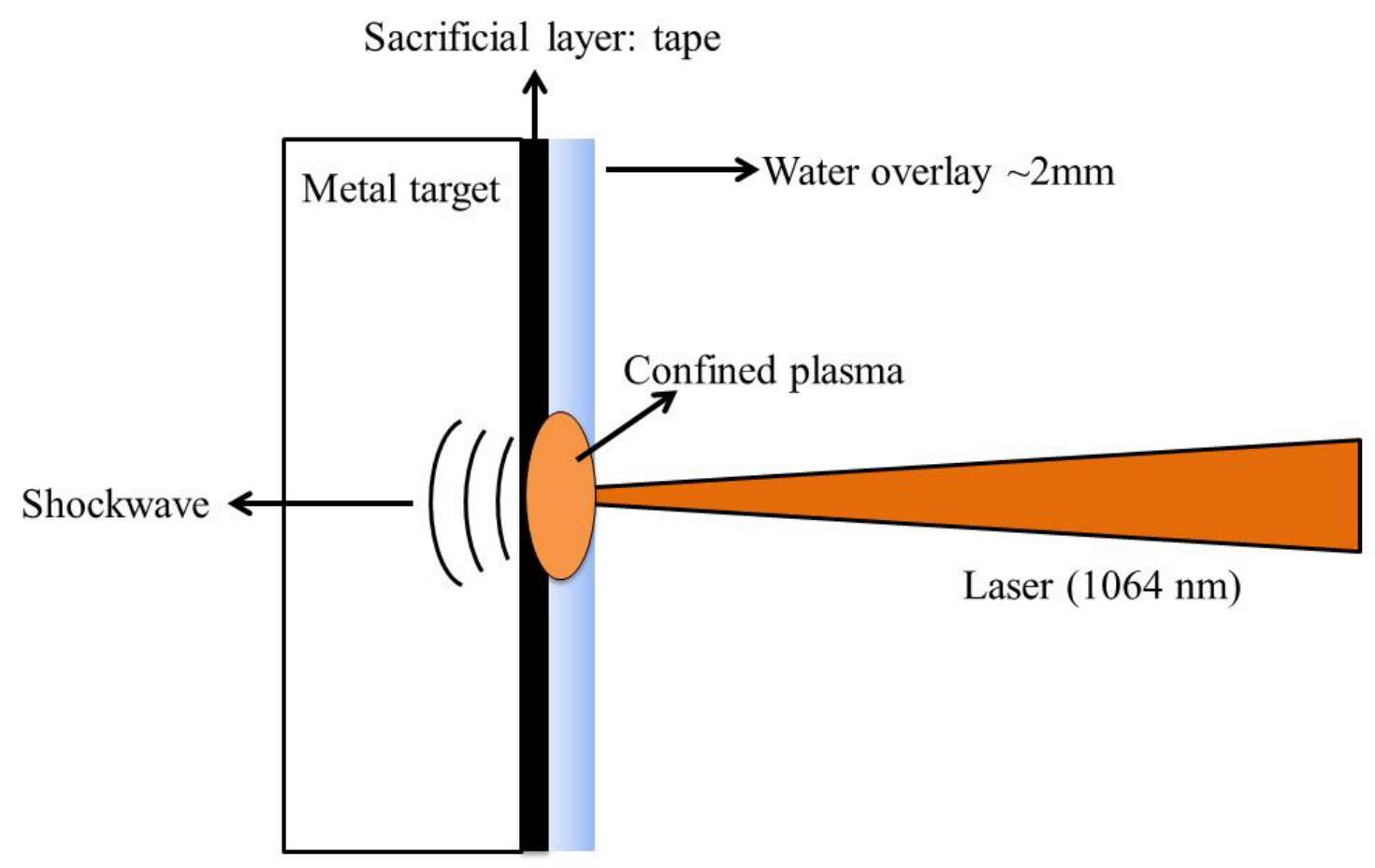

Figure 1. Schematic of the Laser Shock Peening process
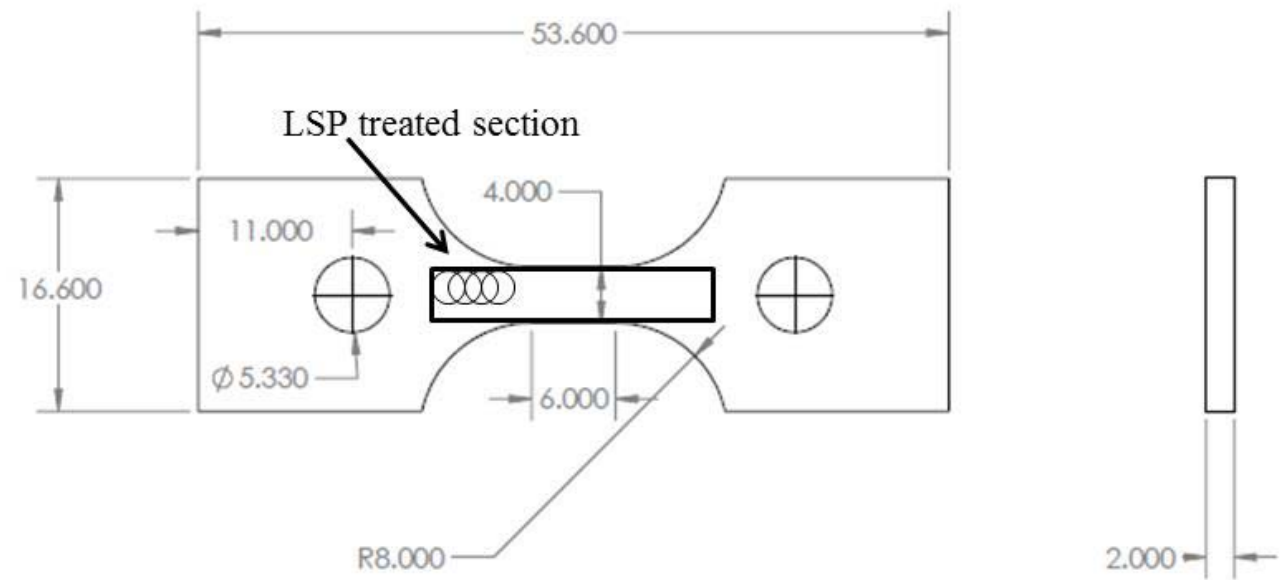

Figure 2. Schematic of the sample used for slow strain rate tests and constant load tests. All dimensions in mm. 

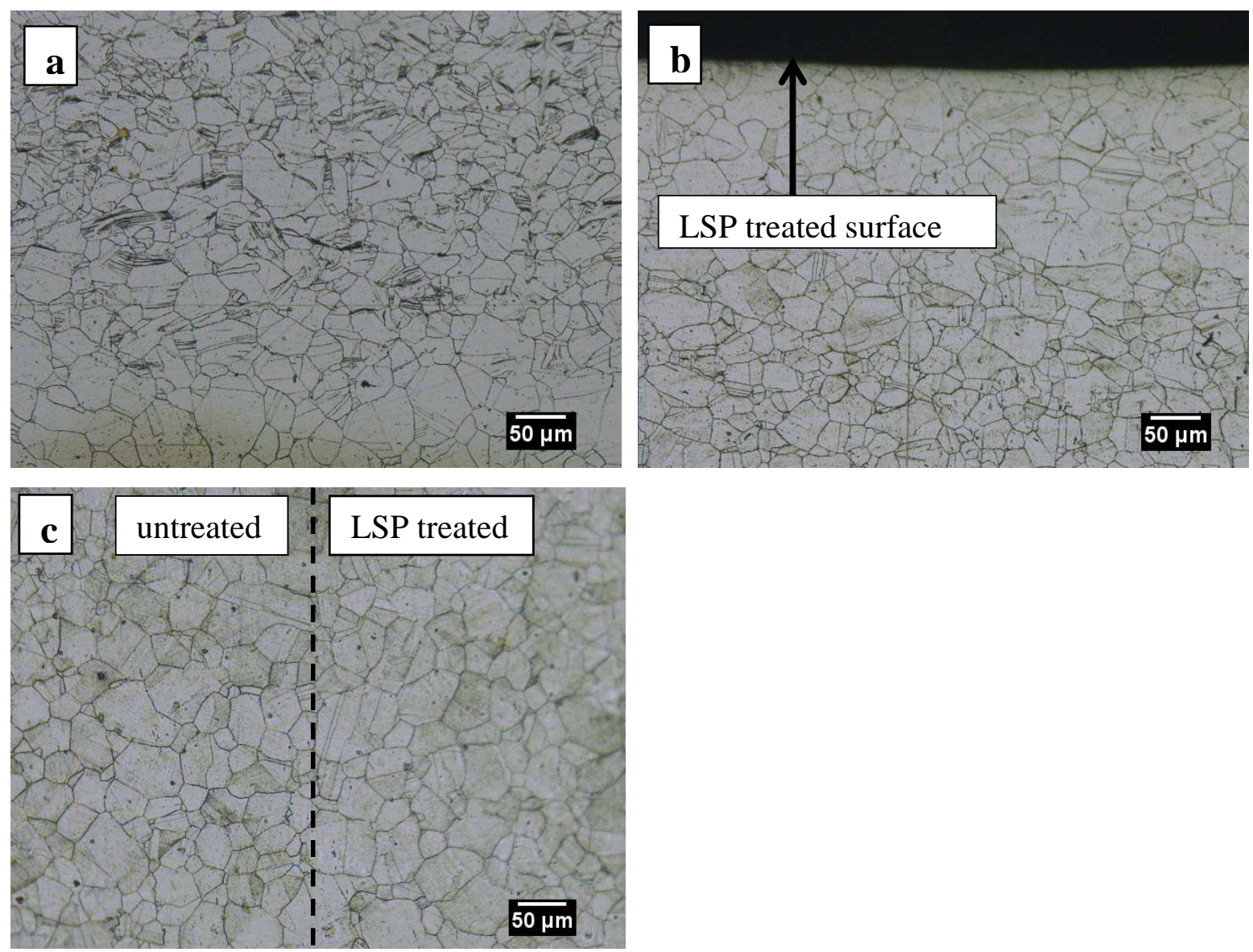

Figure 3. Optical micrographs from cross sections of sensitized a) S1, b) LSP1 (LSP treated) condition samples and c) untreated and LSP treated (right) regions from surface of S1. 

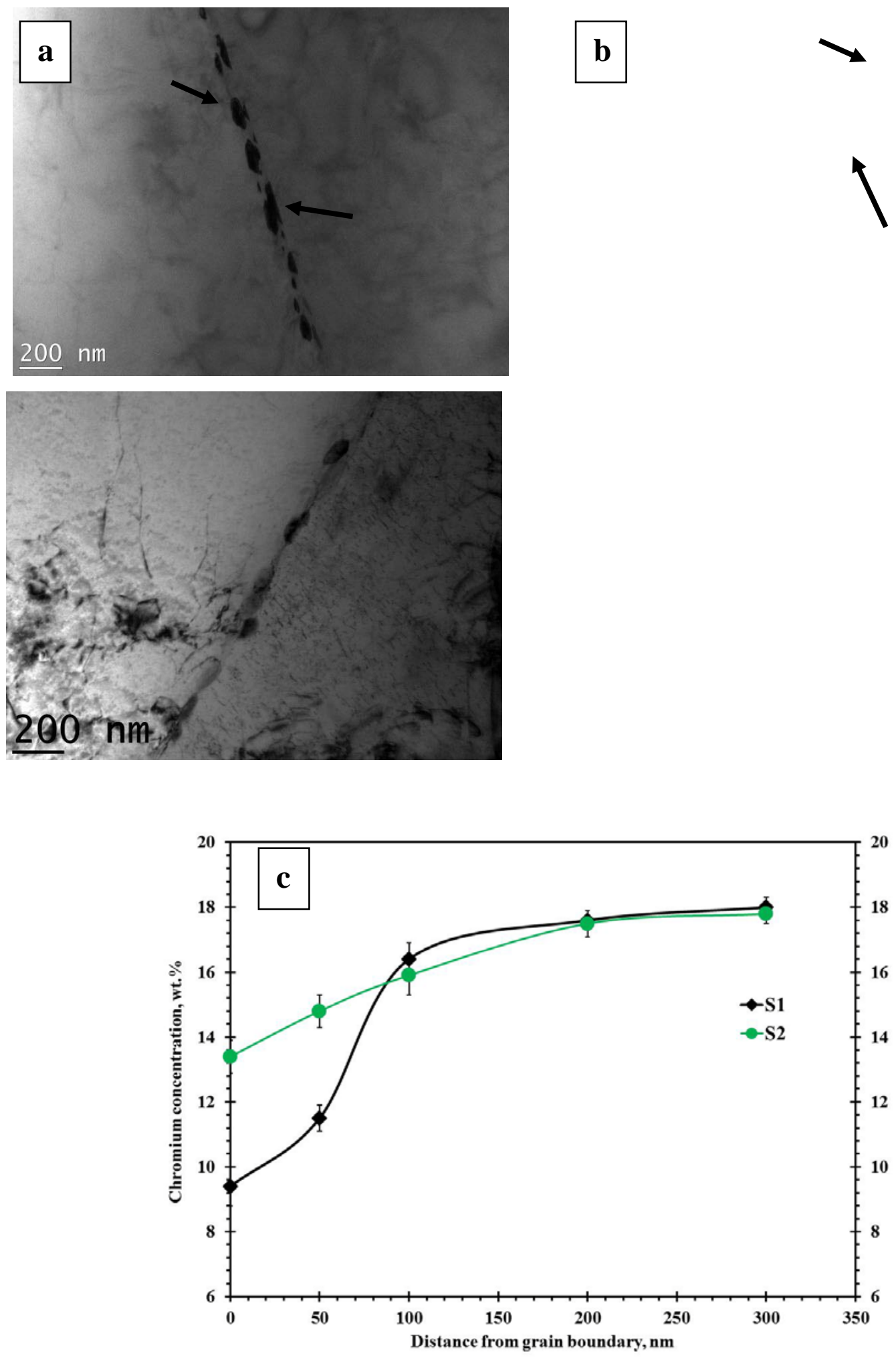

Figure 4. Bright field TEM micrographs showing the presence of carbides along grain boundaries from (a) S1 and (b) S2 condition samples. Chromium concentration profiles at and with distance into the matrix from grain boundaries in S1 and S2 is shown in (c). 

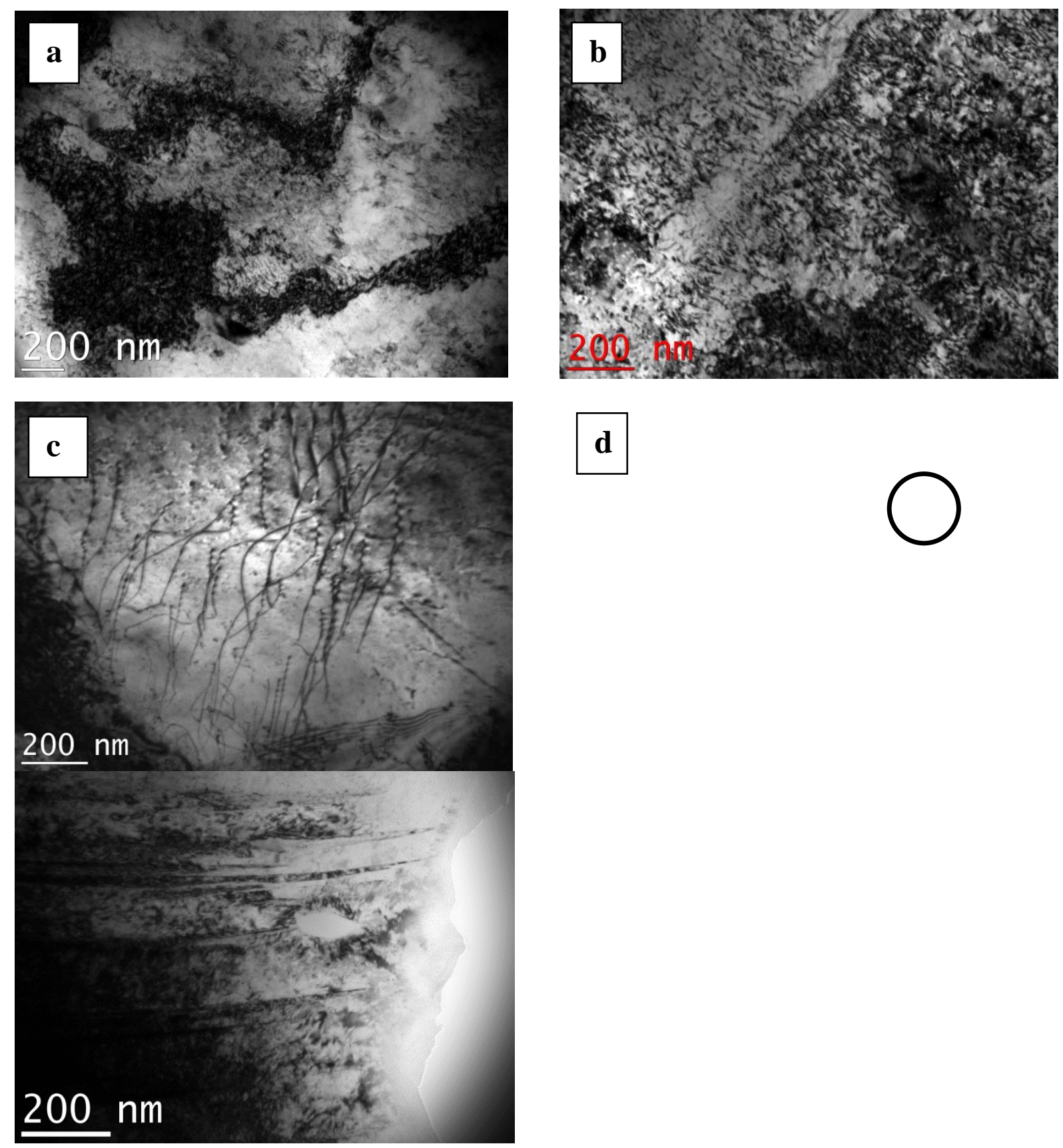

d

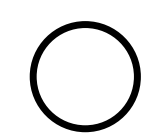



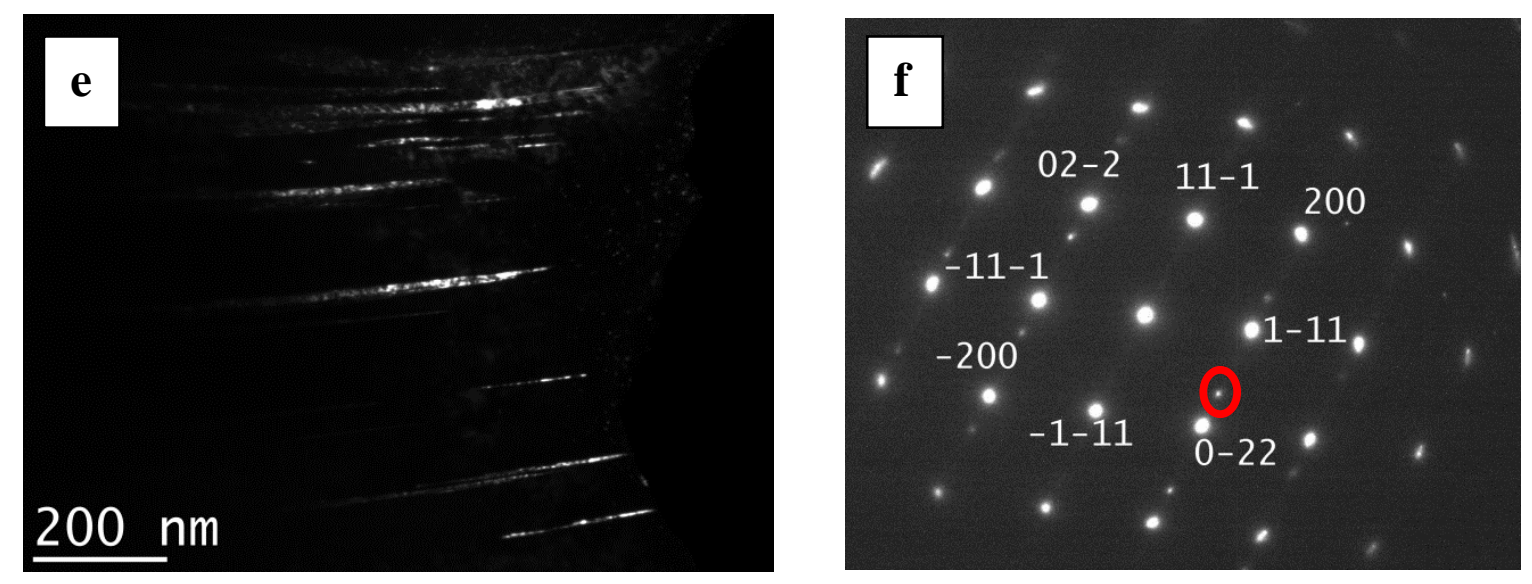

Figure 5. TEM Micrographs from LSP1 sample (a, b and c) showing high dislocation density and 30-200 $\mathrm{nm}$ size sub-grains/crystallites. (d) Bright field micrograph revealing twins, which are confirmed by the extra reflections at the expected positions for twins in FCC crystals in the [011] SAD pattern in (e) from the encircled region in (d). (f) Dark field micrograph recorded from the twin reflection encircled in red in (e) revealing narrow twins. 


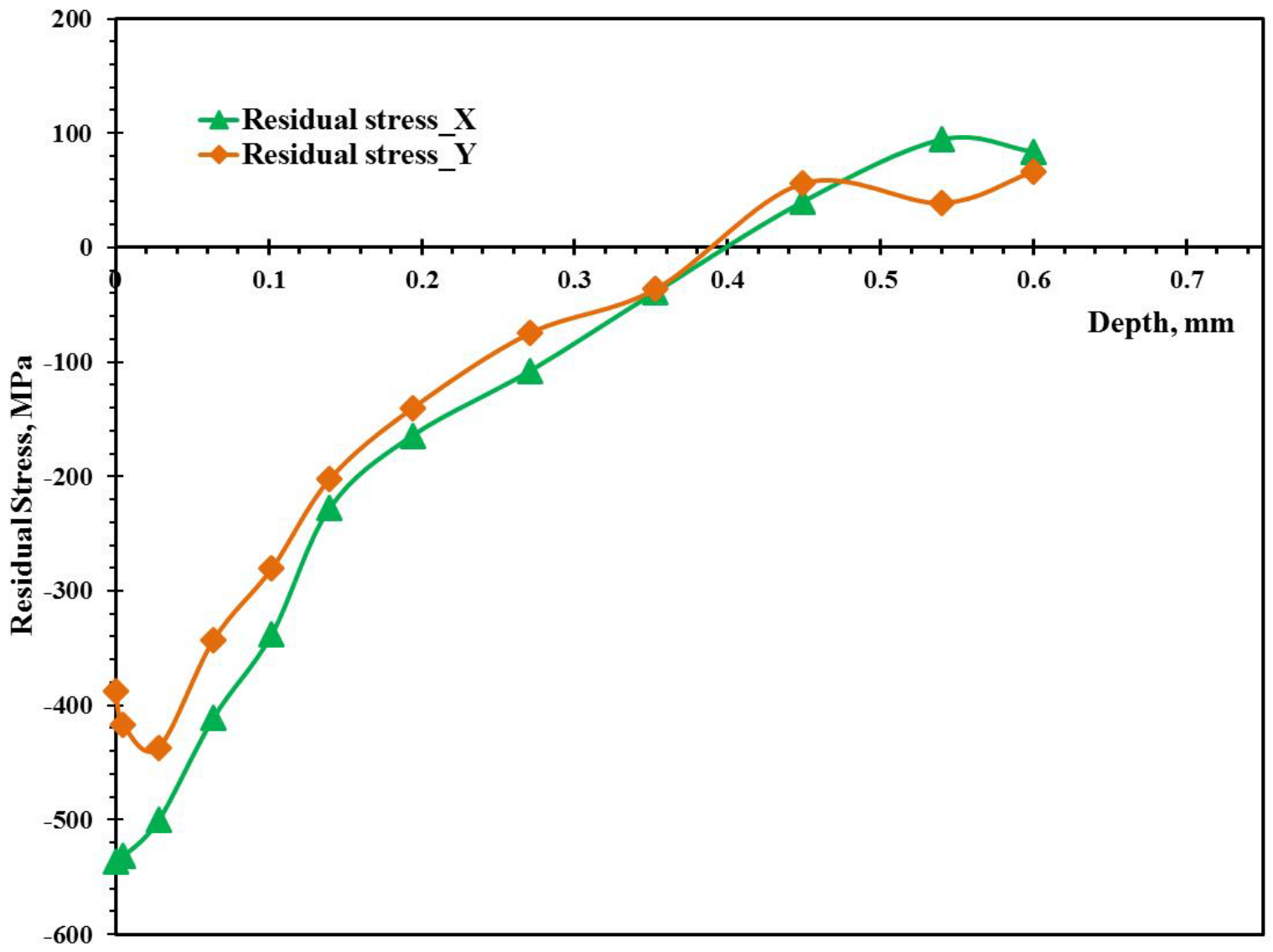

Figure 6. Residual stresses through depth in two orthogonal directions in LSP treated Alloy 600.

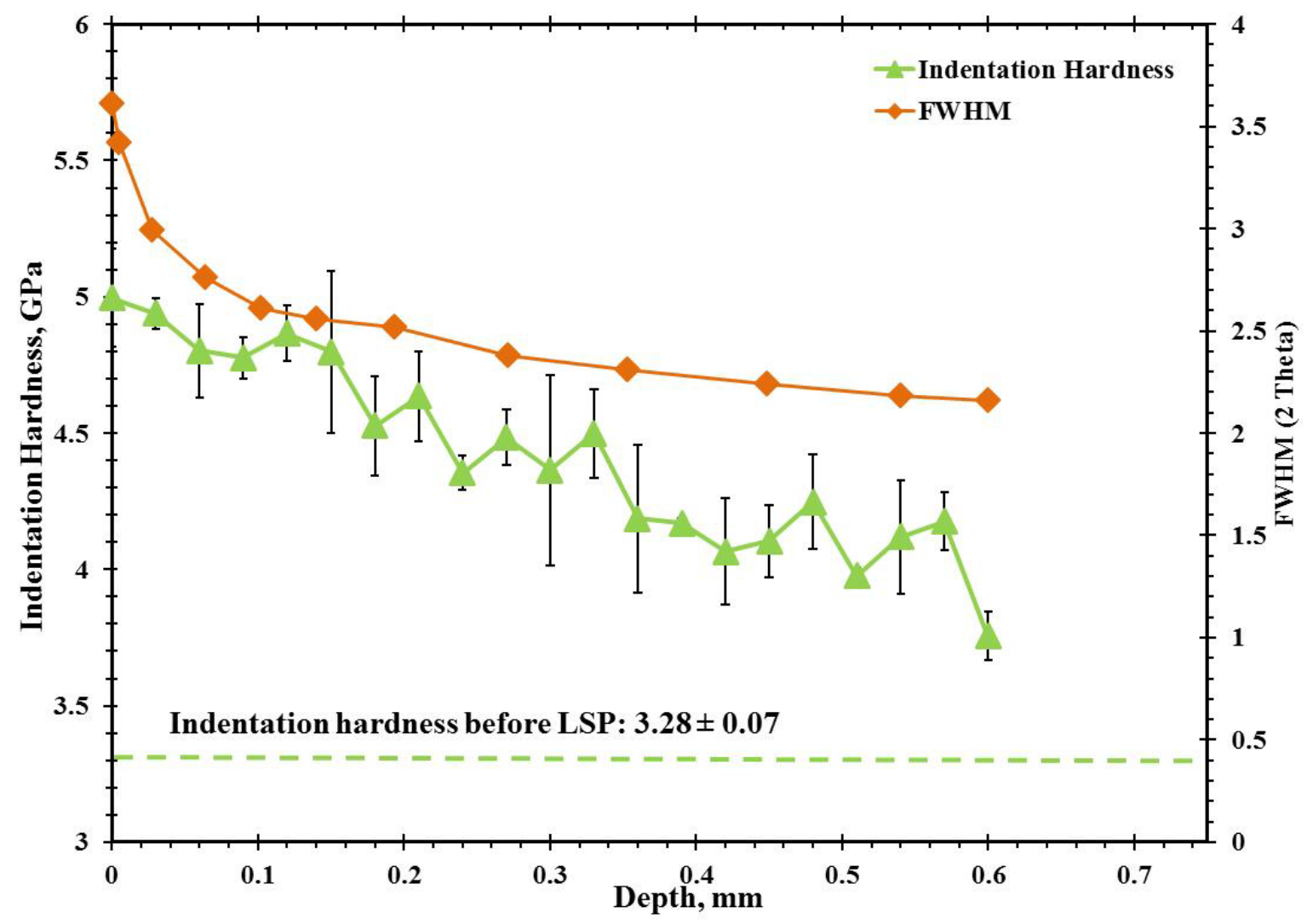


Figure 7. Nano-indentation hardness and FWHM of diffraction peak through depth in LSP treated Alloy 600
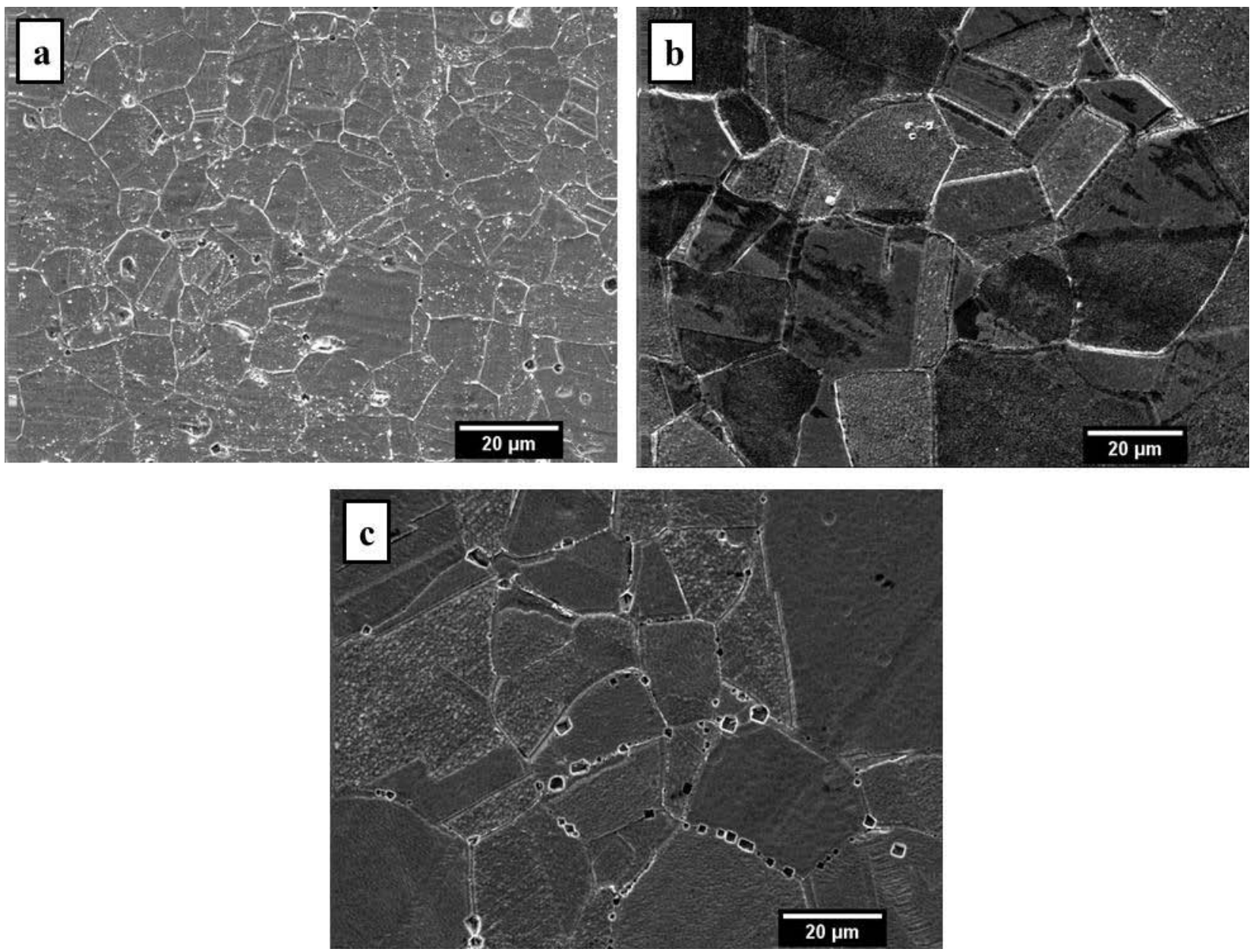

Figure 8. SEM micrographs showing surface microstructure after DLEPR test for a) As received (AR), b) S1 and c) S2 conditions 


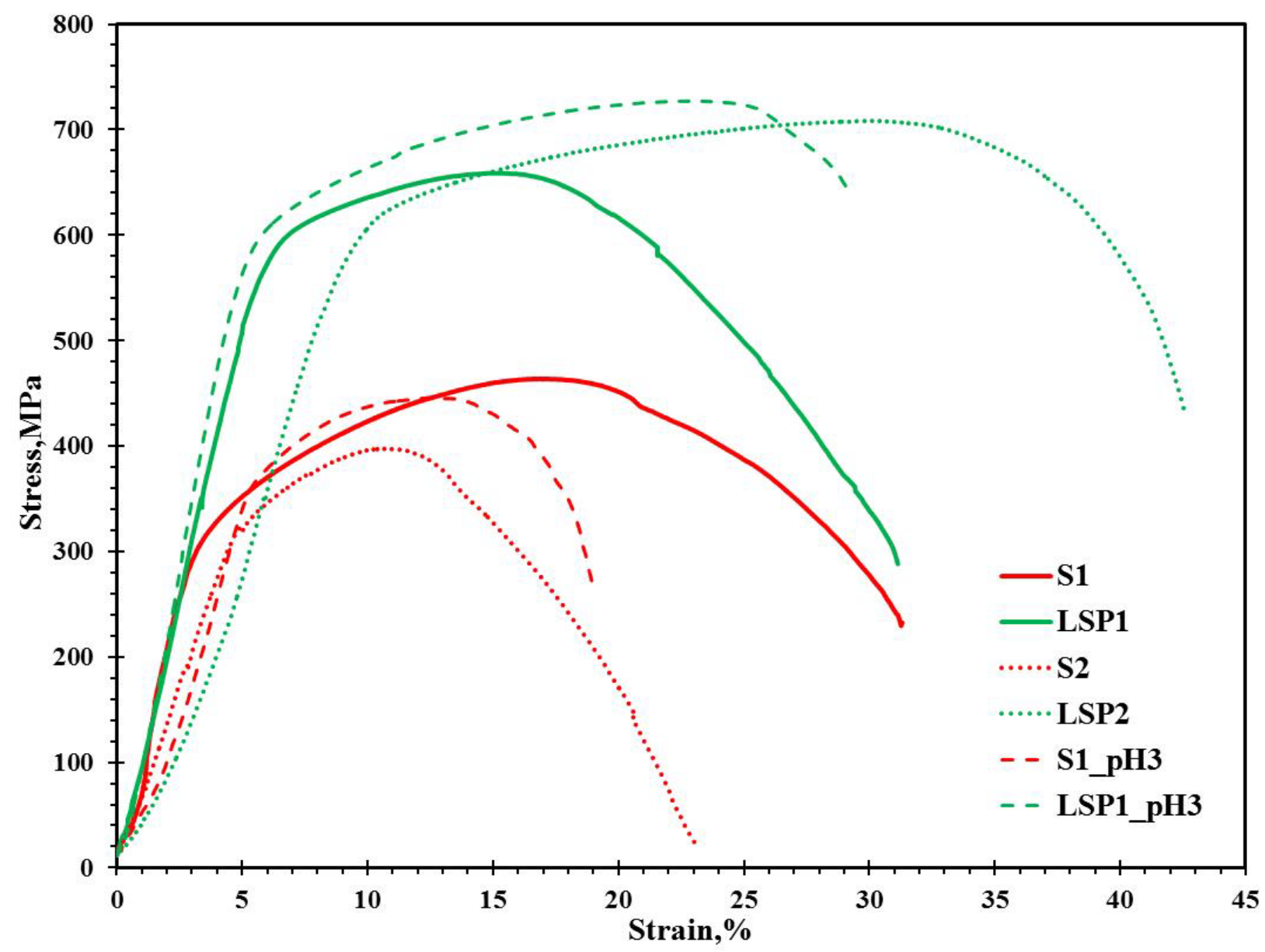

Figure 9. Stress-strain curves of sensitized and LSP treated Alloy 600 tested in $0.001 \mathrm{M}$ tetrathionate solution at room temperature. Strain rate for S1, LSP1 was $2 \times 10^{-6} / \mathrm{s}$ while strain rate for S2, LSP2 was $10^{-6} / \mathrm{s}$. For tests designated with pH3, appropriate amount of $\mathrm{H}_{2} \mathrm{SO}_{4}$ was added to reduce the $\mathrm{pH}$ to 3 . 


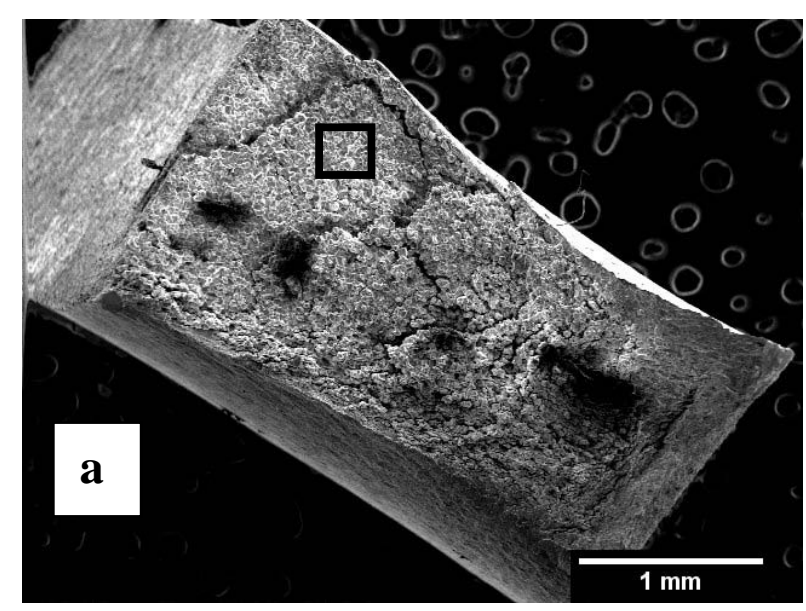

b

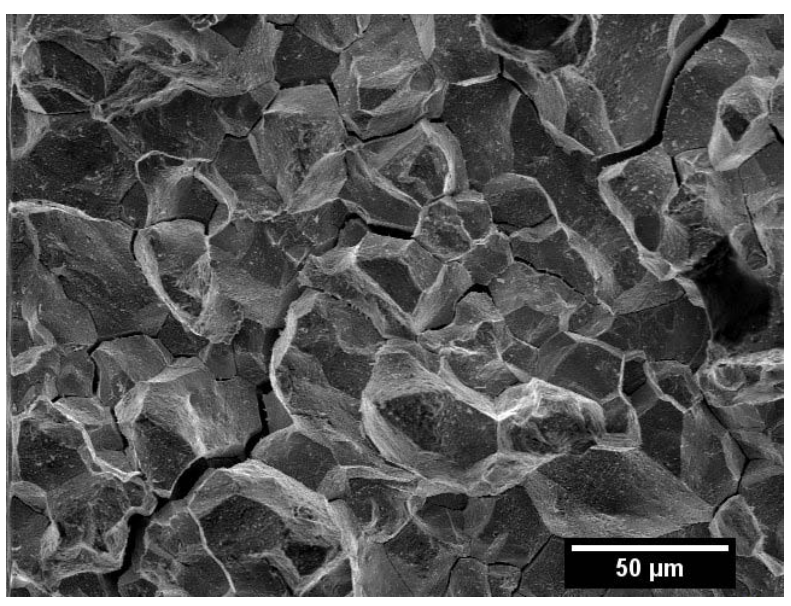



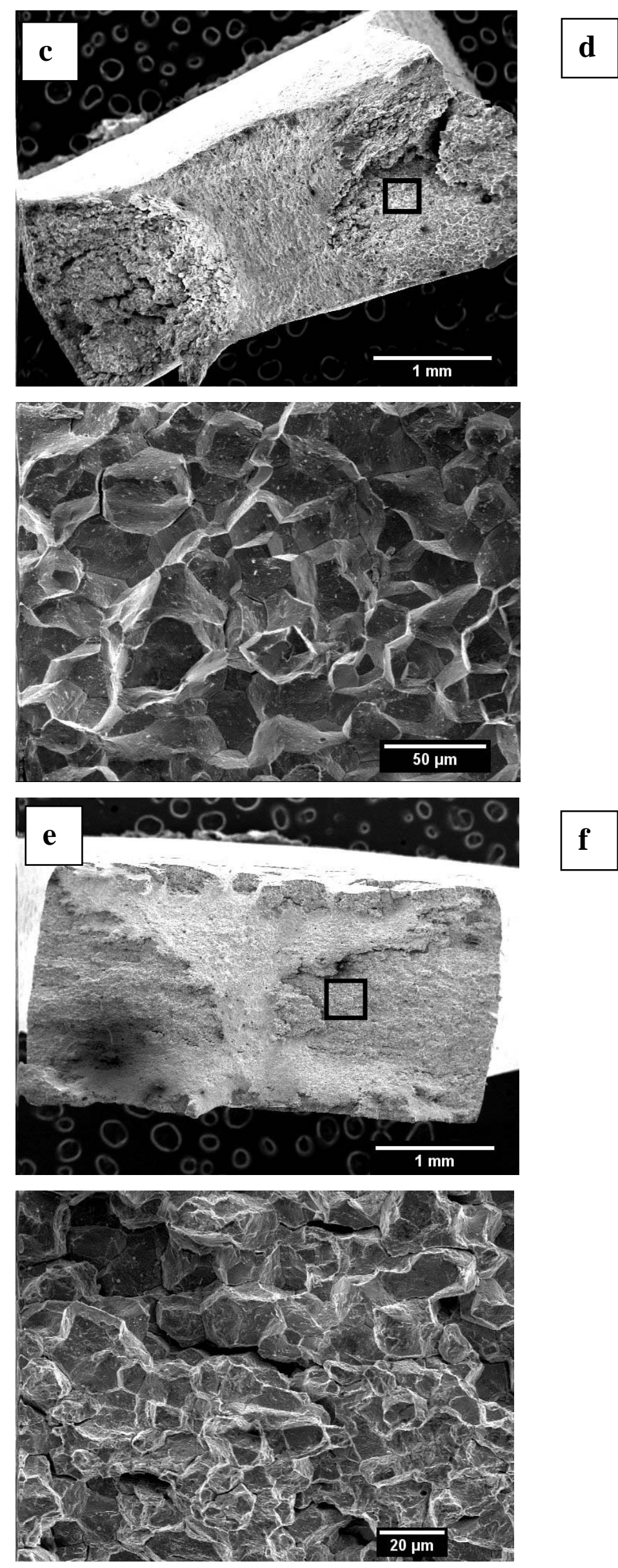

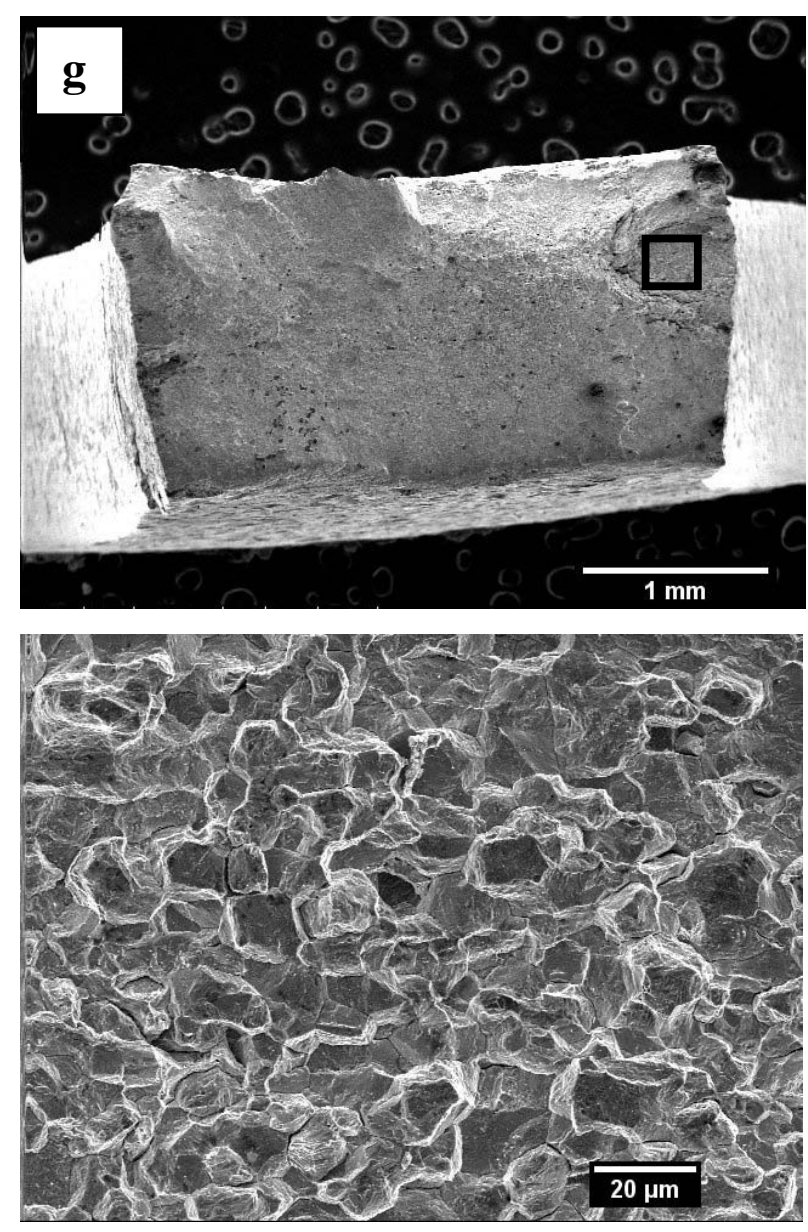

Figure 10. SEM micrographs showing fracture surface from a), b) S1; c), d) LSP1; e), f) S1_pH3 and g), h) LSP1_pH3. Environment: $0.001 \mathrm{M} \mathrm{Na}_{2} \mathrm{~S}_{4} \mathrm{O}_{6}$, pH3 denotes acidified $0.001 \mathrm{M} \mathrm{Na}_{2} \mathrm{~S}_{4} \mathrm{O}_{6}$. Strain rate: $2 \times 10^{-6} \mathrm{~s}^{-1}$. Black boxes in the low magnification SEM micrographs in (a), (c), (e) and (g) highlight the locations for corresponding high magnification micrographs in (b), (d), (f) and (h) and white arrows point to intergranular cracks. 


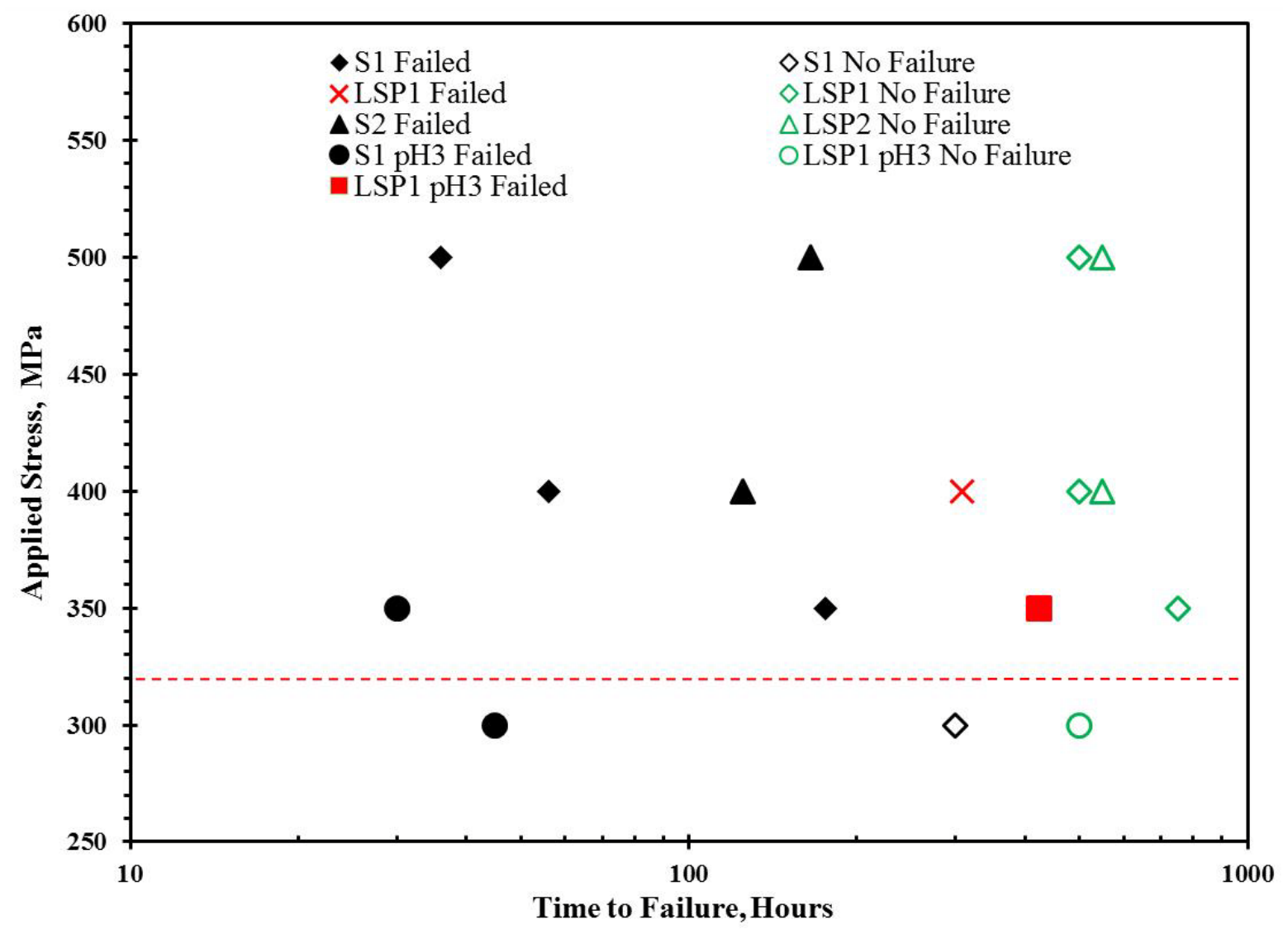

Figure 11. Summary of results of constant load SCC tests on sensitized and LSP treated Alloy 600 samples in $0.001 M \mathrm{Na}_{2} \mathrm{~S}_{4} \mathrm{O}_{6}$ (tetrathionate) solution. Open and closed symbols indicate samples that did not fail in 500 hours and those that failed, respectively. pH3 indicates acidified $0.001 \mathrm{M}$ tetrathionate solution and the red line denotes the yield stress for sensitized Alloy 600 . 


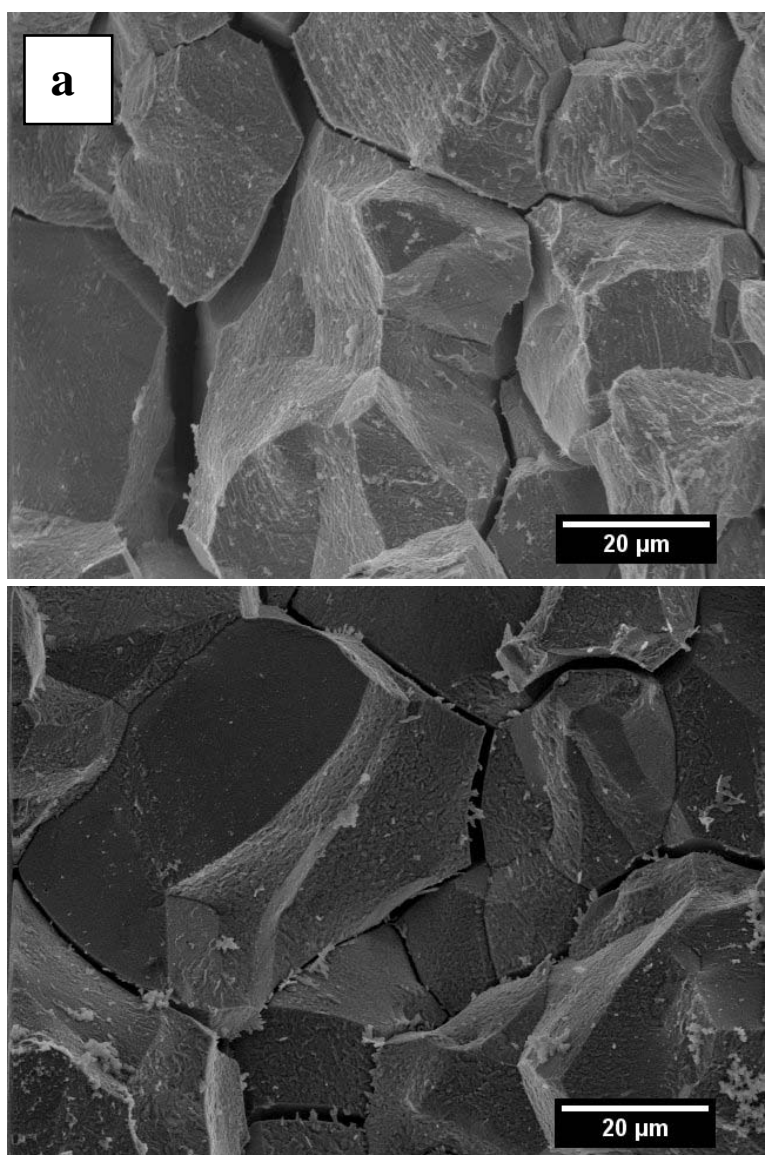

b

Figure 12. SEM micrographs of fracture surface showing intergranular cracking after constant load test (applied stress $=400 \mathrm{MPa}$ ) in $0.001 \mathrm{M}$ tetrathionate solution of a) sensitized (S1) and b) LSP treated (LSP1) Alloy 600 samples. 